$05,11,04$

\title{
Поведение кобальтовой и редкоземельной подсистем в фрустрированных кобальтитах $\mathrm{DyBaCo}_{4} \mathrm{O}_{7+x}$
}

\author{
(C) З.А. Казей ${ }^{1}$, В.В. Снегирев ${ }^{1}$, М.С. Столяренко ${ }^{1}$, К.С. Пигальский ${ }^{2}$, Л.П. Козеева ${ }^{3}$, \\ М.Ю. Каменева ${ }^{3}$, А.Н. Лавров ${ }^{3}$ \\ ${ }^{1}$ Московский государственный университет им. М.В. Ломоносова, \\ Москва, Россия \\ ${ }^{2}$ Институт химической фризики им. Н.Н. Семенова РАН, \\ Москва, Россия \\ ${ }^{3}$ Институт неорганической химии им. А.В. Николаева СО РАН, \\ Новосибирск, Россия \\ E-mail: kazei@plms.phys.msu.ru
}

(Поступила в Редакцию 3 июля 2018 г.)

\begin{abstract}
Проведены экспериментальные исследования структурных, упругих и магнитных свойств кобальтитов $\mathrm{DyBaCo}_{4} \mathrm{O}_{7+x}$, полученных по различной технологии и отличающихся избытком кислорода $x$. Обнаружено, что ромбическое искажение кристаллической структуры в отожженных стехиометрических образцах с $x=0$ приводит к снятию фрустраций и возникновению аномалий упругих свойств в области $T_{N}$, обусловленных упорядочением кобальтовой подсистемы. При незначительном отклонении от стехиометрии по кислороду в закаленных образцах искажение структуры не происходит и аномалии упругих характеристик в области $T_{N}$ размываются и пропадают. Исследования магнитных свойств $\mathrm{DyBaCo}_{4} \mathrm{O}_{7+x}$ показывают, что Р3-подсистема остается парамагнитной и вклад от нее более чем на порядок превышает вклад от Со-подсистемы с сильными антиферромагнитными взаимодействиями. Магнитная восприимчивость стехиометрического образца не обнаруживает заметной аномалии при $T_{N}$, так как упорядочение кобальтовой подсистемы не сопровождается появлением заметного эффективного поля на Р3-ионах.
\end{abstract}

Работа частично выполнена при поддержке субсидии ИХФ РАН в рамках государственного задания ФАНО РФ (тема 45.22 № 0082-2018-0003, АAАA-A18-118012390045-2).

DOI: 10.21883/FTT.2019.01.46896.187

\section{1. Введение}

Окисные соединения кобальта остаются объектами интенсивных экспериментальных и теоретических исследований благодаря их необычному магнитному поведению. Способность катионов кобальта принимать различное зарядовое и спиновое состояния создает широкие возможности для получения новых сложных составов с различными формулами и кристаллическими структурами. Соединения, в которых ионы кобальта занимают различные кристаллографические позиции, позволяет изучать актуальные проблемы физики магнитных явлений: спиновый кроссовер, переход металл-диэлектрик, нетривиальные основные состояния при наличии фрустрации обменных взаимодействий и др.

Слоистые редкоземельные (Р3) кобальтиты $R \mathrm{BaCo}_{4} \mathrm{O}_{7+x} \quad(R=$ редкоземельный ион, $\mathrm{Ca}, \mathrm{Y})$, обнаруживающие фрустрации обменных взаимодействий и смешанную валентность в кобальтовой подсистеме [1-6] и имеющие взаимодействующие $3 d$-кобальтовую и $4 f$-редкоземельную магнитные подсистемы, позволяют изучать такие проблемы магнетизма, как нетривиальные основные магнитные состояния и эффекты ближнего порядка в фрустрированных системах, магнитные переходы при наличии конкурирующих обменных взаимодействий.
Кристаллическая структура $3 d$-кобальтов при высоких температурах описывается гексагональной $P 6_{3} m c$ пространственной группой [7-9] Для некоторых кобальтитов ( $\left.\mathrm{YBaCo}_{4} \mathrm{O}_{7}, \mathrm{TmBaCo}_{4} \mathrm{O}_{7}\right)$ используется тригональная группа $P 31 c[10,11]$, являющаяся подгруппой группы $P 6_{3} m$, что не играет принципиальной роли для большинства физических характеристик. Для стехиометрических соединений $R \mathrm{BaCo}_{4} \mathrm{O}_{7}$ с трехвалентным редкоземельным ионом $R^{3+}$ ионы кобальта имеют смешанную валентность $\mathrm{Co}^{2+}$ и $\mathrm{Co}^{3+}$ в соотношении $3: 1$ и распределены в структуре неупорядоченно по двум типам тетраэдрических позиций. В каркасной кристаллической структуре тетраэдры $\mathrm{CoO}_{4}$, объединенные общими углами, образуют два вида слоев, треугольные (Т) слои и слои Кагоме $(\mathrm{K})$, которые упакованы поочередно вдоль оси $c$ (рис. $1, a$ ). При этом катионы $R^{3+}$ и $\mathrm{Ba}^{2+}$ занимают более крупные октаэдрические и кубооктаэдрические позиции, соответственно, в трехмерной тетраэдрической сетке.

Группа из ближайших ионов кобальта представляет собой бипирамиду с треугольным основанием из ионов Со2 в $K$-слое и ионов Co1 в вершинах из $T$-слоев, при этом все расстояния между ближайшими ионами Co1-Co2 сравнимы по величине (лежат в пределах $3.2-3.3 \AA$ ) (рис. $1, b)$. В гексагональной структуре ио- 


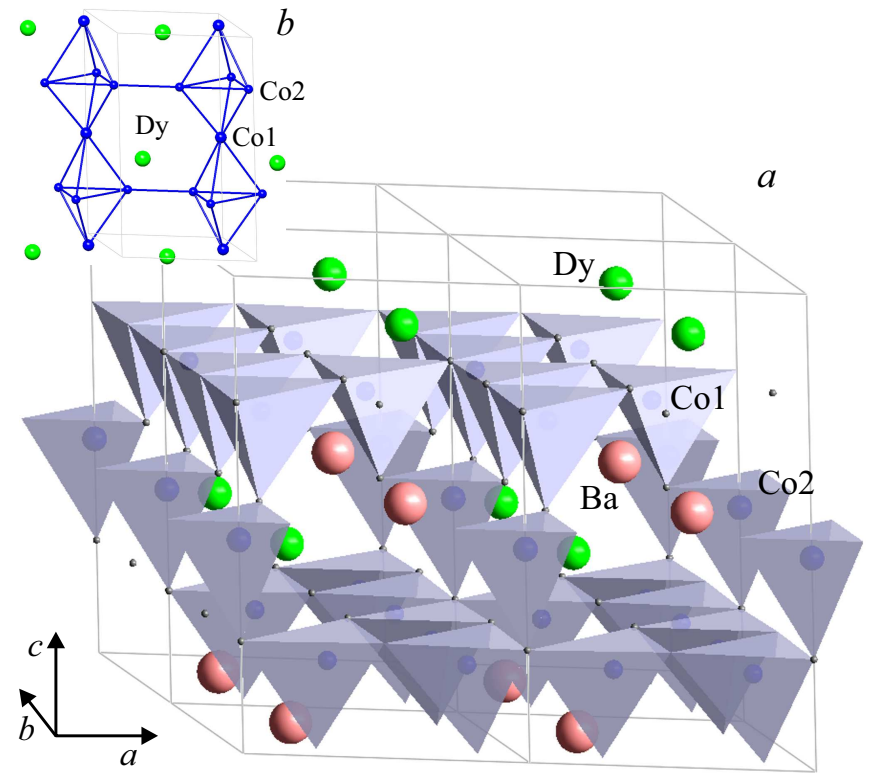

Рис. 1. Общий вид гексагональной кристаллической структуры $\mathrm{DyBaCo}_{4} \mathrm{O}_{7}$ (показаны только катионы); ионы кобальта Co1 и $\mathrm{Co} 2$ внутри кислородных тетраэдров в $K$ и $T$ слоях изображены шариками различного размера $(a)$. Сетка магнитных ионов $\mathrm{Co} 1$ (средние шарики) и $\mathrm{Co} 2$ (маленькие шарики) в структуре $(b)$.

ны Co2, образующие треугольники в $K$-слоях, связаны эквивалентным сильным антиферромагнитным взаимодействием $J_{1}$ (рис. $\left.1, b\right)$. Взаимодействие $J_{2}$ этих ионов с ионами Co1 в двух соседних $T$-слоях (апикальные ионы), по-видимому, сравнимо по величине с параметром $J_{1}$ в плоскости и приводит к образованию цепочек обменно связанных спинов вдоль оси $c$. За счет ромбического искажения структуры возникают неэквивалентные по величине обменные взаимодействия внутри $K$-слоя и между слоями.

Двумерная решетка Кагоме, состоящая из соединенных углами треугольников, хорошо известна как структурная топология, приводящая к геометрической фрустрации. Фрустрации в магнитной системе часто приводят к вырожденному основному состоянию, а также к отсутствию дальнего магнитного порядка даже при значительных константах обменного взаимодействия [12]. Искажение кристаллической структуры способно частично или полностью снять фрустрации и благоприятствует появлению дальнего магнитного порядка [13].

Слоистые редкоземельные кобальтиты $R \mathrm{BaCo}_{4} \mathrm{O}_{7+x}$ образуются с тяжелыми Р3-ионами, начиная с Tb. Соотношение разновалентных ионов $\mathrm{Co}^{2+} / \mathrm{Co}^{3+}$ в кобальтовой подсистеме можно варьировать с помощью неизовалентного замещения $R^{3+} \rightarrow \mathrm{Ca}^{2+}$ или $\mathrm{Co}^{2+} / \mathrm{Co}^{3+} \rightarrow \mathrm{Zn}^{2+} / \mathrm{Al}^{3+}$, а также изменением содержания кислорода $(7+x)$. Это дает возможность изучать влияние средней валентности в Со-подсистеме на фазовые переходы и физические свойства этих фрустрированных соединений.

Наиболее изученным в этом семействе является соединение с немагнитным ионом Y, которое позволяет исследовать поведение кобальтовой подсистемы. Эксперименты по нейтронной дифракции Ү-кобальтита показывают, что при $T_{S}=313 \mathrm{~K}$ он испытывает структурный переход, сопровождающийся понижением симметрии от тригональной до орторомбической (пространственная группа $\left.\mathrm{Pbn} 2_{1} ; a_{0} \approx a_{h}, b_{0} \approx \sqrt{3} a_{h}\right)$. Величина ромбического искажения невелика и составляет $\varepsilon_{0}=\left(a_{0}-b_{0} / \sqrt{3}\right) / a_{0}=-4.0 \cdot 10^{-3} \quad[10]$. За счет структурного перехода геометрические фрустрации в $\mathrm{YBaCo}_{4} \mathrm{O}_{7}$ снимаются и ниже $T_{S}$ возникает трехмерное антиферромагнитное упорядочение [1], сопровождающееся ниже $T_{N}$ понижением симметрии до моноклинной $P 112_{1}$ [12,14]. О величине обменного взаимодействия в кобальтовой подсистеме этого семейства можно судить по значению парамагнитной температуры $\theta_{C W}=-508 \mathrm{~K}$ в законе Кюри-Вейсса для магнитной восприимчивости $\mathrm{YBaCo}_{4} \mathrm{O}_{7}$ [1]. Из-за фрустрированности обменных взаимодействий дальний магнитный порядок, однако, устанавливается при существенно более низкой температуре $T_{N} \sim 110 \mathrm{~K}$ и характеризуется сложной неколлинеарной магнитной структурой $[1,12]$. При дальнейшем понижении температуры наблюдаются еще один магнитный фазовый переход при $T_{N 2} \sim 70 \mathrm{~K}$ [15], который обусловлен спиновой переориентацией в Со-подсистеме и не приводит к дальнейшему понижению симметрии [12].

Для других Р3-кобальтитов с магнитными Р3-ионами структура также обнаруживает небольшое искажение, происходящее в виде фазового перехода, сопровождаемого аномалиями упругих, магнитных и транспортных свойств. Критическая температура структурного перехода $T_{S}$ монотонно повышается при увеличении радиуса P3-иона и для ионов Но-Тb, Y лежит выше комнатной температуры. Это искажение структуры при фазовом переходе в Р3-кобальтитах снимает фрустрацию обменных взаимодействий, что сказывается на магнитных фазовых переходах в Со-подсистеме, которые имеют место при $T_{N}<T_{S}[13,16]$.

В разных работах, посвященных исследованию Р3-кобальтитов, имеются противоречивые данные о магнитной конфигурации, основном состоянии, температурах и особенностях структурных и магнитных фазовых переходов. Одной из причин подобных расхождений, возможно, является использование в экспериментах образцов с различным содержанием кислорода [1]. Влияние отклонение кислородного индекса от стехиометрического значения на фазовые переходы в Р3-кобальтитах и их физические свойства практически не исследованы. Наши исследования системы $\mathrm{YBaCo}_{4} \mathrm{O}_{7+x}$ показали, что при небольшом отклонении от стехиометрии структура остается гексагональной неискаженной, фрустрации сохраняются и как следствие магнитный переход в Со-подсистеме быстро размывается (если вообще сохраняется переход с дальним магнитным порядком) [17]. В насто- 
ящей работе исследуется влияние нестехиометрии по кислороду на структурные и упругие характеристики, а также на магнитные фазовые переходы в кобальтитах $\mathrm{DyBaCo}_{4} \mathrm{O}_{7+x}$, полученных по разной технологии и имеющих различное отклонение от стехиометрии. Ионные радиусы для ионов Dу и Y достаточно близки, так что кристаллическая структура и обменные параметры для двух систем, по-видимому, также близки. Поэтому представляют интерес сравнительные исследования кобальтитов с ионами Y и Dy, имеющих одинаковое отклонение от стехиометрии. Открытым остается также вопрос о поведении Р3-подсистемы и о взаимодействии Р3- и Со-подсистем в этих двух подрешеточных магнетиках сложного состава.

\section{2. Образцы и экспериментальная техника}

Исследования структурных и упругих свойств проводились на поликристаллических образцах $\mathrm{DyBaCo}_{4} \mathrm{O}_{7+x}$ $(x=0-0.1)$, синтезированных по керамической технологии. Твердофазный синтез проводили на воздухе из оксидов $\mathrm{Dy}_{2} \mathrm{O}_{3}$ (ИтО-МГр.), $\mathrm{Co}_{3} \mathrm{O}_{4}$ (99.7\%, Alfa-Aesar) и карбоната $\mathrm{BaCO}_{3}$ (ос. ч.), предварительно отожженных при температурах 800,700 и $400^{\circ} \mathrm{C}$ соответственно. Стехиометрическую смесь исходных компонентов тщательно перетирали в агатовой ступке, используя ацетон или этанол, загружали в корундовый тигель, уплотняли и помещали в муфельную печь. В данной работе синтез проводили в три стадии при температурах 900, 1000 и $1100^{\circ} \mathrm{C}$ (время отжига при каждой температуре $20 \mathrm{~h}$ ) с промежуточным перетиранием. Каждая стадия завершалась закалкой образца от $900-950^{\circ} \mathrm{C}$ [18]. Перед последним отжигом при $1100^{\circ} \mathrm{C}$ порошок прессовался в таблетки диаметром $15 \mathrm{~mm}$ и толщиной $3 \mathrm{~mm}$.

Содержание кислорода в керамических образцах $\mathrm{DyBaCo}_{4} \mathrm{O}_{7+x}$ измерялось весовым методом с разрешением $\Delta x \approx 0.001$ и абсолютной точностью \pm 0.01 , определяющейся привязкой к двум реперным составам $x=0$ и $x=1.50$. После синтеза и закалки от $900-950^{\circ} \mathrm{C}$, содержание кислорода в таблетках керамики лежит в диапазоне $x \sim 0.05-0.10$, в зависимости от морфологии керамики и параметров закалки. Для получения требуемого содержания кислорода и его однородного распределения по объему образцы керамики подвергались дополнительной термообработке (обозначаются в тексте $A$ ), имеющей свою специфику по сравнению с другими оксидами. Ступенчатая зависимость равновесного содержания кислорода от температуры $x_{\text {eq }}(T)$ в $\mathrm{DyBaCo}_{4} \mathrm{O}_{7+x}$ [19] не позволяет получать требуемые значения $x$, используя методику отжига до достижения равновесия при заданных значениях температуры и парциального давления кислорода.

$\mathrm{B} \mathrm{DyBaCo}_{4} \mathrm{O}_{7+x}$ промежуточные составы с различным избытком кислорода $0<x<1.5$ и однородным его распределением по объему удается получить, лишь используя зависимость $x(t)$ от времени отжига и понизив температуру настолько, что вхождение кислорода лимитируется поверхностным обменом. Массивные образцы керамики должны иметь достаточно высокую пористость для равномерного доступа кислорода к зернам керамики. В данной работе использовалась керамика с плотностью $\sim 75-80 \%$ от рентгеновской. Содержание кислорода $x=0$ было получено отжигом в вакууме при $500^{\circ} \mathrm{C}$, а значение $x=0.10$ - отжигом в течение нескольких суток на воздухе при $T=210^{\circ} \mathrm{C}$. Для сравнения исследовался также образец, закаленный от 900-950 ${ }^{\circ} \mathrm{C}$ и не подвергавшийся дополнительной термообработке. Этот образец, обозначенный в тексте $Q$, имеет интегральное содержание кислорода $x=0.07$, как будет показано далее.

Рентгенографические исследования проводились на дифрактометре Stoe с монохроматором на первичном пучке $\left(\mathrm{Co} K_{\alpha 1}\right.$ излучение). Все линии на рентгенограммах исследуемых образцов $\mathrm{DyBaCo}_{4} \mathrm{O}_{7+x}$ при комнатной температуре индицировались в рамках гексагональной или слабо искаженной гексагональной структуры. Некоторые образцы содержали небольшое количество (до 3\%) оксида диспрозия. Полнопрофильный анализ рентгенограммы в интервале углов $(18-120)^{\circ}$ с использованием программы Full_Prof позволил определить значения параметров $a, b$ и $c$ искаженной элементарной ячейки (обозначения для орторомбической ячейки) и их изменение при изменении кислородного индекса.

Модуль Юнга $E$ и коэффициент внутреннего трения $q^{-1}$ измерялись методом составного резонатора на частоте $\sim 110 \mathrm{kHz}$ в интервале температур $(80-280) \mathrm{K}$. В автоматизированной установке в качестве задающего генератора использовался генератор Agilent 33120A, в качестве регистрирующего устройства - цифровой осциллограф Tektronix TDS 1002 с дополнительным усилителем SR 560. Температура измерялась и контролировалась с помощью температурного контроллера Lakeshore 331 с точностью $\pm 0.03 \mathrm{~K}$. Температурные измерения проводились в режиме стационарного состояния, а температурный шаг и выдержка в области фазового перехода варьировались в широких пределах, обеспечивающих стационарность. Управление экспериментом и первичная обработка проводилась на стандартном IBM совместимом компьютере с интерфейсом IEEE-488 с использованием оригинального программного обеспечения. Из экспериментальной амплитудночастотной характеристики $A(f)$ в области резонанса методом математической обработки получалась сглаженная кривая $A_{s m}(f)$ и определялись амплитуды и частоты резонанса и антирезонанса (ширина окна $\Delta f$ при сглаживании варьировалась в зависимости от качества исходных кривых $A(f)$ ). Температурные зависимости амплитуд и частот резонанса и антирезонанса позволяли определять изменение модуля Юнга и поглощения (коэффициента внутреннего трения) в широком диапазоне температур. 
Измерения намагниченности были выполнены индукционным методом в импульсных магнитных полях до $280 \mathrm{kOe} \mathrm{(время} \mathrm{возрастания} \mathrm{поля} \mathrm{в} \mathrm{импульсе} \mathrm{со-}$ ставляло $\sim 3 \mathrm{~ms})$. В течение импульса поля с интервалом $0.4 \mu$ s проводилась цифровая запись интегрированных сигналов с измерительной $M(t)$ и полевой $H(t)$ катушек. Сигнал раскомпенсации измерительной катушки записывался в аналогичных условиях в отсутствие образца и вычитался при программной обработке экспериментальных данных. Эта обработка позволяла получить сглаженные функции намагниченности $M(H)$ и ее производной $d M(H) / d H$. Начальная магнитная восприимчивость определялась как из наклона линейного участка кривых намагничивания в полях до $50 \mathrm{kOe}$, так и из прямых измерений на переменном токе $\chi_{a c}(T)$. Магнитная восприимчивость в слабом низкочастотном магнитном поле (амплитуда $10 \mathrm{Oe}$, частота $980 \mathrm{~Hz}$ ) измерялась по двух катушечной методике. Образец в форме длинного цилиндра помещался в измерительную катушку, рядом с которой располагалась компенсационная катушка, имеющая близкие параметры. С интервалом $\sim 20 \mu$ s производилась оцифровка разностного и опорного сигналов с катушек. Действительная и мнимая компоненты магнитной восприимчивости рассчитывались при численной обработке полученных данных. Чувствительность методики измерения восприимчивости составляла $\sim 10^{-5}$ СГСМ.

\section{3. Экспериментальные результаты и обсуждение}

\section{1. Рентгеноструктурный анализ}

Рентгеновские исследования позволяют обнаружить искажения структуры, которые снимают фрустрации обменных взаимодействий, и таким образом влияют на магнитное упорядочение в кобальтовой подсистеме. Структура соединений $\mathrm{DyBaCo}_{4} \mathrm{O}_{7+x}$ исследовалась при комнатной температуре на специально приготовленных отожженных образцах $A$ с $x=0,0.1$ и закаленном образце $Q$. Рентгенограммы исследованных образцов при комнатной температуре обнаруживают существенное отличие.

Далее приводится часть рентгенограммы в интервале углов $(55-70)^{\circ}$, которая содержит рефлексы, чувствительные как к ромбическому искажению, так и к наличию двухфазного состояния образца. Полнопрофильный анализ рентгенограммы нестехиометрического образца c $x=0.1$ показывает, что он имеет неискаженную гексагональную структуру с параметрами $a_{h}=6.3048 \AA$, $c_{h}=10.2365 \AA$ (рис. 2, $a$; индексы для всех фаз приводятся в орторомбической установке $h=h_{0}=h_{h}+k_{h}$, $k=k_{0}=h_{h}-k_{h}, l=l_{0}=l_{h}$, таблица). Ширина линии $w(2 \theta)$ меняется от 0.1 до $0.2^{\circ}$ в диапазоне углов $(18-120)^{\circ}$, что типично для замещенных составов, в которых некоторый параметр, в нашем случае избыток

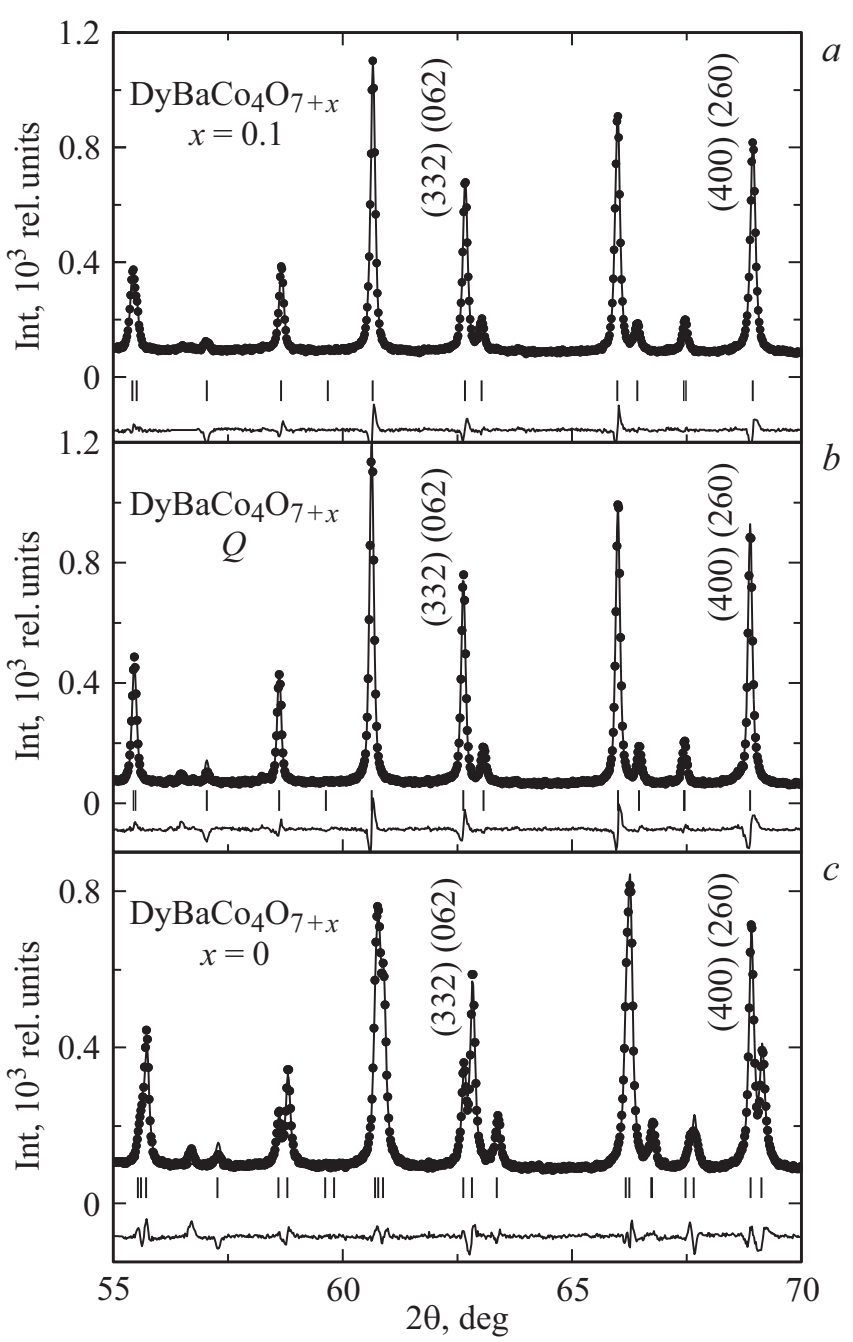

Рис. 2. Экспериментальные (точки) и рассчитанные (линии) рентгенограммы и разность между ними (линии внизу) для образцов $\mathrm{DyBaCo}_{4} \mathrm{O}_{7+x}$ с разным отклонением от стехиометрии: $x=0.1(a), x=0(c)$, и закаленного образца $Q(b)$ на $\mathrm{Co} K_{\alpha 1}$ излучении. Штрихами показаны положения брэгговских пиков согласно пространственной группе.

кислорода $x$, несколько варьируется по объему. Такого типа нестехиометрические соединения с ионами Y не обнаруживают структурного перехода вплоть до гелиевых температур $[14,17]$. Полно профильный анализ рентгенограммы закаленного образца $Q$ показывает, что он также имеет неискаженную гексагональную структуру с очень близкими параметрами $a_{h}=6.3097 \AA, c_{h}=10.2311 \AA$ (рис. 2, b, таблица).

На рентгенограмме стехиометрического образца c $x=0$ наблюдается заметное отличие для ряда рефлексов, чувствительных к ромбическому искажению, например рефлексов $\{(332)+(062)\}$ и $\{(400)+(260)\}$ с углами $2 \theta \approx 63$ и $69^{\circ}$ (рис. $2, c$ ). Действительно, структура этого образца описывается в первом приближении с учетом небольшого ромбического искажения, величина которого при обработке полной рентгенограммы в 
Параметры $a\left(a_{a v}\right.$ для искаженного образца $), b(\sqrt{3} a$ для гексагонального образца $), c$ элементарной ячейки, величина орторомбического искажения $\varepsilon_{0}$ образцов $\mathrm{DyBaCo}_{4} \mathrm{O}_{7+x}$, полученных по разной технологии с различным избытком кислорода $x$ (параметр $\chi^{2}$ характеризует качество описания рентгенограммы)

\begin{tabular}{l|c|c|c|c|c}
\hline Образец, $x$ & $a\left(a_{a v}\right), \AA$ & $b(\sqrt{3} a), \AA$ & $c, \AA$ & $\varepsilon_{0}, 10^{-3}$ & $\chi^{2}$ \\
\hline 0 & 6.3061 & 10.9676 & 10.2193 & -4.14 & 2.71 \\
0.1 & $(6.3191)$ & $(10.9203)$ & 10.2365 & 0 & 3.22 \\
$\mathrm{Q}$ & 6.3048 & $(10.9288)$ & 10.2311 & 0 & 3.92
\end{tabular}

диапазоне углов $(18-120)^{\circ}$ составляет $\varepsilon_{0}=-4.1 \cdot 10^{-3}$. Величина искажения, определенная по отдельным рефлексам, также лежит вблизи этого значения.

Расщепление рефлекса $\{(400)+(260)\}$, наиболее чувствительного к ромбическому искажению в указанной области углов, напрямую дает величину искажения $\varepsilon_{0}=2\left(d_{1}^{2}-d_{2}^{2}\right) /\left(4 d_{1}^{2}-d_{2}^{2}\right),\left(d_{1,2}=2 \sin \left(\theta_{1,2}\right) / \lambda\right)$, где $\theta_{1}=\theta_{400}, \theta_{2}=\theta_{260}$ - брэгговские углы соответствующих рефлексов. Видно, что для образца с $x=0$ с небольшим орторомбическим искажением рефлекс обнаруживает расщепление на две линии (рис. 2,c), в то время как нестехиометрическому образцу с $x=0.1$ и закаленному образцу $Q$ соответствует нерасщепленный рефлекс для $d_{1}=d_{2}$ при $b_{0}=a_{0} \sqrt{3}$ (рис. 2, $a, b$ ). Отметим, что соотношение интенсивностей $I_{1} / I_{2}=2: 1$ расщепленных линий для искаженного образца соответствуют статистическому значению для такого типа искажения. При этом три оси типа $\langle h 00\rangle$ или $\langle h h 0\rangle$ в гексагональной установке становятся неэквивалентными, что приводит к расщеплению соответствующих пиков на две компоненты с соотношением интенсивностей $2: 1$ или $1: 2$ в зависимости от знака искажения.

Значения средних параметров ячейки для искаженного $a_{a v}=(a+b / \sqrt{3}) / 2, c$ и неискаженных образцов, согласно полнопрофильному анализу, близки $a_{a v} \approx a_{h}$, $c \approx c_{h}$ (см. табл.). Из сравнения параметров решетки для двух структур видно, что при отклонении $x=0.1$ параметр $c$ увеличивается на $0.017 \AA$, а параметр $a_{a v}$ уменьшается на $0.014 \AA$. Предполагая линейную зависимость параметров ячейки $a_{a v}$, и $c$ от параметра $x$ можно оценить для закаленного образца $Q$ отклонение от стехиометрии $x \approx 0.07$. Качество описания рентгенограммы закаленного образца $Q$ хуже, чем для искаженного образца $A$ с $x=0$, о чем можно судить по величине параметра $\chi^{2}$ и разностной функции, приведенной внизу каждой из панелей рис. 2. Это может свидетельствовать либо о расслоении на фазы, либо о неоднородном по параметру $x$ составе образца. Возможно, учет наличия второй искаженной фазы позволит несколько уменьшить величину $\chi^{2}$. Склонность кобальтитов к образованию двухфазного состояния (расслоение на фазы) при отклонении образца от стехиометрии наблюдалась и обсуждалась ранее для систем $\mathrm{YbBaCo}_{4} \mathrm{O}_{7+x}[10]$ и $\mathrm{YBaCo}_{4} \mathrm{O}_{7+x}[17]$.

\section{2. Модуль Юнга и внутреннее трение}

Исследования упругих свойств позволяют регистрировать температуру перехода и изучать влияние на переход различных факторов. Наши исследования показали, что модуль Юнга Р3-кобальтов в области структурного перехода обнаруживает резкий минимум, тем более выраженный, чем ниже температура $T_{S}$. При этом характер аномалии в области $T_{N}$ для серии образцов $\mathrm{YBaCo}_{4} \mathrm{O}_{7+x}$ $(x=0,0.1,0.2)$ в очень сильной степени определяется стехиометрией [17]. В данной работе исследовались температурные зависимости модуля Юнга $E(T)$ и внутреннего трения $q^{-1}(T)$ серии соединений $\mathrm{DyBaCo}_{4} \mathrm{O}_{7+x}$, полученных по различной технологии и отличающихся величиной искажения структуры и содержанием кислорода. Для удобства сравнения для всех образцов на рисунках приведены относительные изменения модуля $\Delta E(T) / E_{0}\left(\Delta E(T)=E(T)-E_{0} ; E_{0}=E(280 \mathrm{~K})\right)$, нормированные на значение $E_{0}$ при $T=280 \mathrm{~K}$.

Для образцов с различной термообработкой, которые отличаются величиной искажения структуры, температурные зависимости $\Delta E(T) / E_{0}$ обнаруживают различное поведение. В интервале температур (80-280) К кривые $\Delta E(T) / E_{0}$ демонстрируют гистерезис, величина которого, вообще говоря, зависит от предыстории данной серии измерений. Для образца, быстро охлажденного от комнатной температуры до температуры жидкого азота (за $\sim 20 \mathrm{~min}$; закалка), кривая $\Delta E(T) / E_{0}$ сильно отличается, но последующее термоциклирование приводит к стабильной петле, для которой ширина между кривыми при охлаждении и нагреве уже не меняется и определяется свойствами образца: технологией синтеза образцов, содержанием кислорода, искажением структуры. На рисунках далее приводятся и обсуждаются такие установившиеся петли гистерезиса.

Для неискаженного образца $Q$, кривые $\Delta E(T) / E_{0}$ при охлаждении от комнатной температуры обнаруживают нетипичное смягчение $\sim 3 \cdot 10^{-2}$, и значительный гистерезис (рис. 3 , кривые $1^{\prime}$ и $2^{\prime}$ ). При этом величина гистерезиса (ширина петли) составляет $\sim 1.5 \cdot 10^{-2}$. Такое поведение, на наш взгляд, свидетельствует о неравновесном состоянии закаленного образца $Q$ при термоцикировании. Наоборот, для стехиометрического образца $A$ с $x=0$ смягчение на кривых $\Delta E(T) / E_{0}$ не превышает $\sim 1 \cdot 10^{-2}$, а гистерезис практически отсут- 


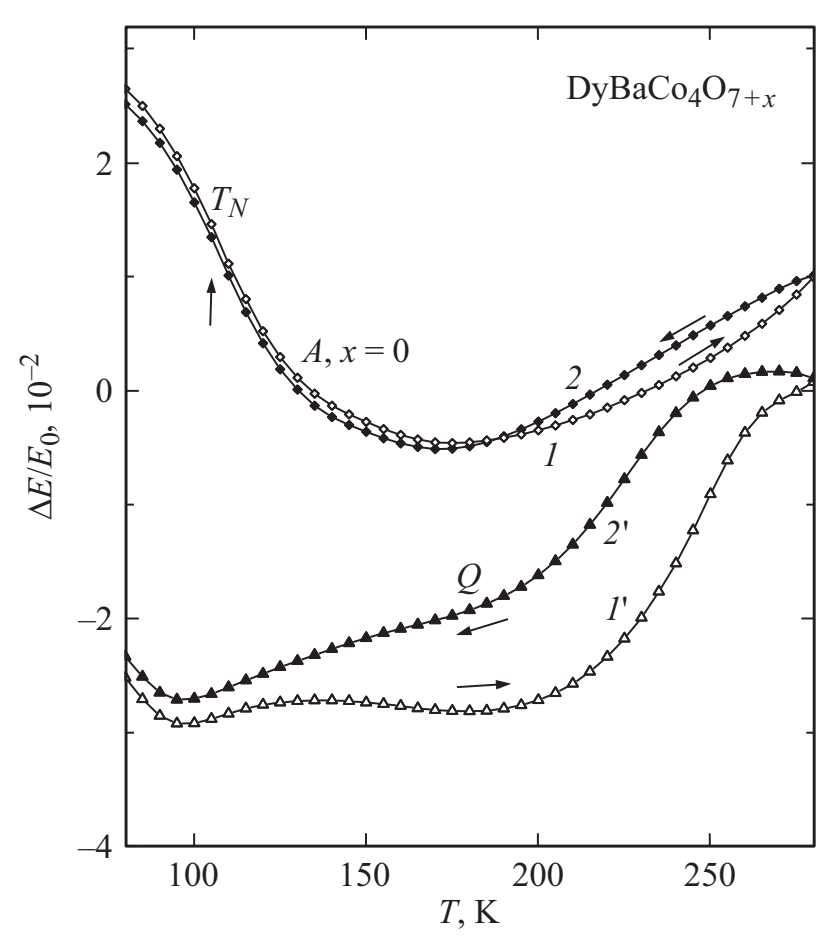

Рис. 3. Зависимость относительной величины модуля Юнга $\Delta E / E_{0}$ от температуры для отожженного $A(x=0)$ и закаленного $Q$ образцов кобальтитов $\mathrm{DyBaCo}_{4} \mathrm{O}_{7+x}$ при нагреве (светлые точки) и охлаждении (темные точки; номера кривых соответствуют последовательности термоциклирования).

ствует. Для исследуемых образцов $A$ и $Q$ прослеживается связь между величинами гистерезиса и результирующего смягчения модуля, которая была обнаружена ранее для серии образцов $\mathrm{YBaCo}_{4} \mathrm{O}_{7+x}$. Отметим, что поведение закаленных образцов $Q$ кобальтитов с ионами Dу и Y близки, тогда как отожженные образцы заметно отличаются.

Исследованные образцы $A$ и $Q$ обнаруживают также качественно разное поведение модуля Юнга в области магнитного фазового перехода. Ранее было подробно исследовано влияние нестехиометрии на магнитный фазовый переход в Со-подсистеме для серии образцов $\mathrm{YBaCo}_{4} \mathrm{O}_{7+x}$, подвергнутых дополнительному отжигу на воздухе. Для стехиометрического искаженного образца с $x=0$ в области магнитного фазового перехода $T_{N} \sim 105 \mathrm{~K}$ на фоне монотонного изменения $\Delta E(T) / E_{0}$ наблюдаются скачки модуля Юнга, имеющие место, как при нагреве, так и при охлаждении, и воспроизводящиеся при повторном термоциклировании. Величины скачков при охлаждении и нагреве практически совпадают и составляют $\delta E\left(T_{N}\right) / E_{0} \approx 1 \cdot 10^{-2}$. Точка фазового перехода $T_{N}$ соответствует скачку на зависимости $\Delta E(T) / E_{0}$ или максимуму производной модуля Юнга по температуpe (рис. 4). Температура магнитного фазового перехода второго рода, определенная по максимуму на кривой $\left(E_{0}^{-1}\right) d E / d T$, совпадает с литературными данными. Температура перехода составляет $T_{N}=(104.0 \pm 0.2) \mathrm{K}$ и заметно не меняется при отклонении от стехиометрии. Для стехиометрического соединения $\mathrm{YBaCo}_{4} \mathrm{O}_{7}$ похожие аномалии наблюдаются и в области второго магнитного фазового перехода с $T_{N 2} \sim 70 \mathrm{~K}$.

При увеличении содержания кислорода в нестехиометрических образцах $\mathrm{YBaCo}_{4} \mathrm{O}_{7+x} \mathrm{c} x=0.1$ и 0.2 скачки на температурных зависимостях модуля Юнга $\Delta E / E_{0}$ при $T_{N}$ очень резко уменьшаются, размываются и становятся практически ненаблюдаемыми. Их следы можно обнаружить только на производной $\left(E_{0}^{-1}\right) d E / d T$, имеющей максимум в области $T_{N}$. Закаленный образец $Q$ кобальтита $\mathrm{YBaCo}_{4} \mathrm{O}_{7+x}$, для которого значение $x \leq 0.1$, также обнаруживает только очень размытый максимум на производной (вставка на рис. 4). Характерно, что для стехиометрического Dу-кобальтита аномалия, соответствующая упорядочению в Со-подсистеме, существенно менее выражена и видна только на производной (рис. 4). Еще менее выраженной становится аномалия на кривых $\left(E_{0}^{-1}\right) d E / d T$ для закаленного образца $Q$ (вставка на pис. 4). Для Еr-кобальтита аномалия в области $T_{N}$ близка по характеру и величине к наблюдаемой аномалии для Ү-кобальтита. Таким образом, величина аномалии зависит как от отклонения от стехиометрии, так и от типа Р3-иона.

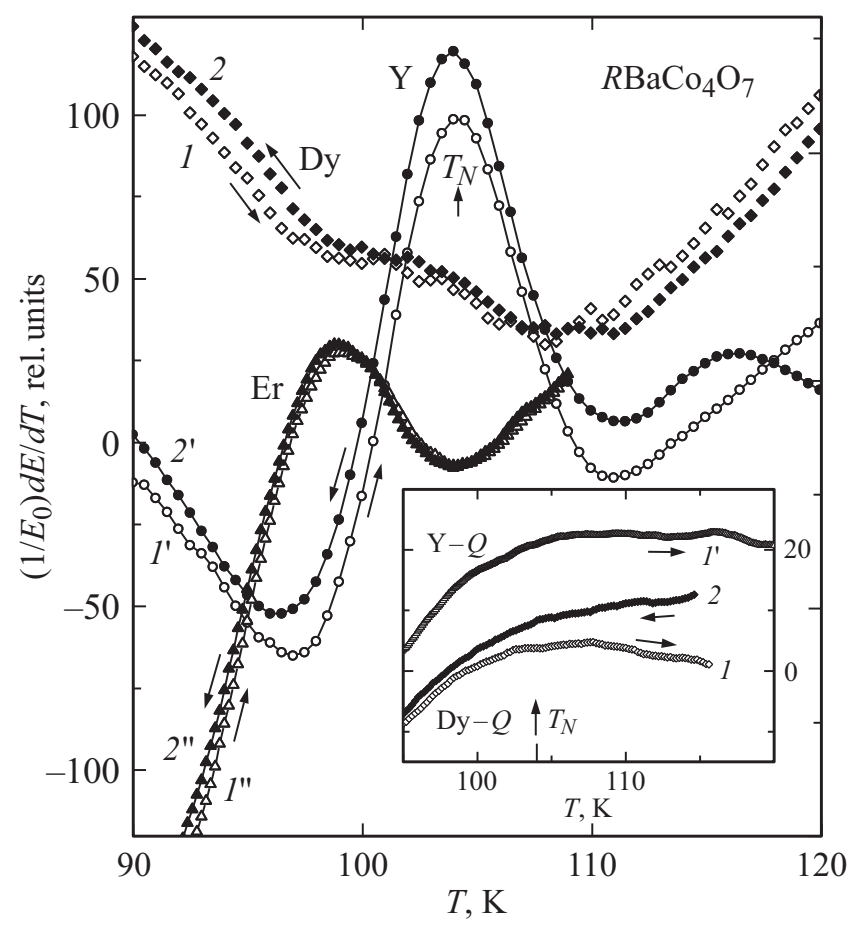

Рис. 4. Температурная зависимость производной модуля Юнга $\left(1 / E_{0}\right) d E / d T$ для стехиометрических образцов $R \mathrm{BaCo}_{4} \mathrm{O}_{7}$ с различными Р3-ионами при нагреве (светлые точки) и охлаждении (темные точки) в области магнитного фазового перехода (кривые $1,2-R=\mathrm{Dy} ; 1^{\prime}, 2^{\prime}-R=\mathrm{Y} ; 1^{\prime \prime}, 2^{\prime \prime}-R=\mathrm{Er}$ ). Кривые для различных $R$ смещены по вертикальной оси на произвольную величину. На вставке показаны зависимости $\left(1 / E_{0}\right) d E / d T$ для закаленных образцов $R \mathrm{BaCo}_{4} \mathrm{O}_{7+x}$ с ионами Dy (кривые 1,2$)$ и Y (кривая $1^{\prime}$ ). 


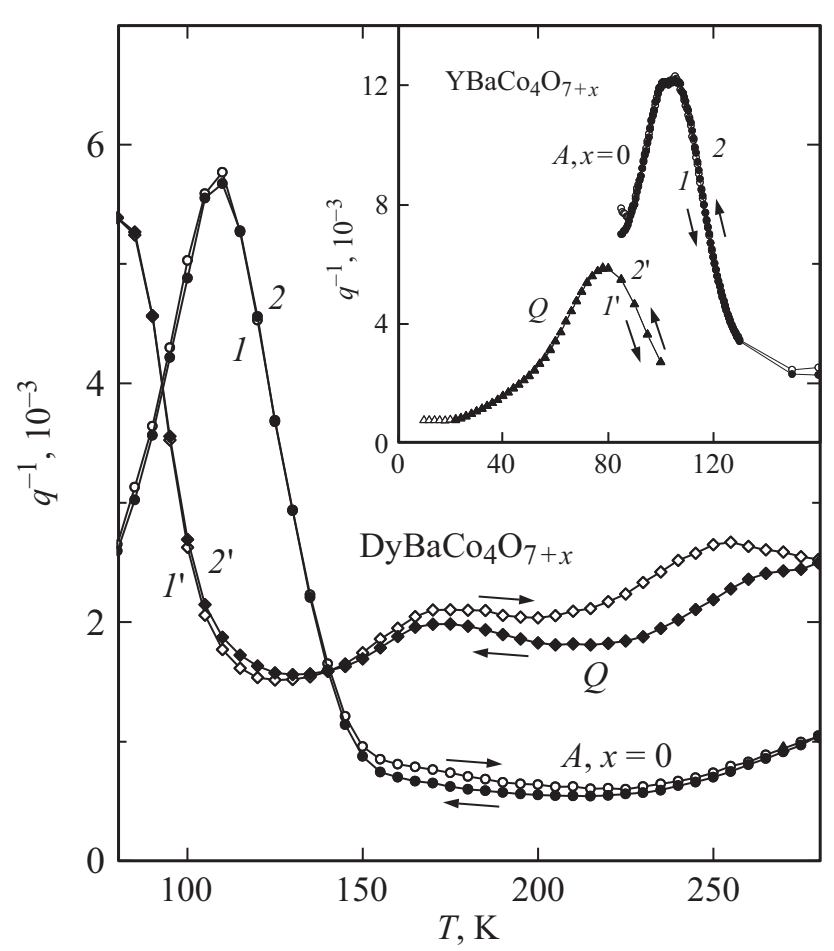

Рис. 5. Температурная зависимость внутреннего трения $q^{-1}$ для отожженного $A(x=0$, кривые 1,2$)$ и закаленного $Q$ (кривые $1^{\prime}, 2^{\prime}$ ) образцов $\mathrm{DyBaCo}_{4} \mathrm{O}_{7+x}$ при нагреве (светлые точки) и охлаждении (темные точки; номера кривых соответствуют последовательности термоциклирования). На вставке показаны зависимости $q^{-1}(T)$ для отожженного $A$ $(x=0$, кривые 1,2$)$ и закаленного $Q$ (кривые $\left.1^{\prime}, 2^{\prime}\right)$ образцов $\mathrm{YBaCO}_{4} \mathrm{O}_{7+x}$.

Влияние нестехиометрии по кислороду на магнитные фазовые переходы в кобальтитах можно объяснить следующим образом. Искажение структуры стехиометрических образцов $R \mathrm{BaCo}_{4} \mathrm{O}_{7+x}$ при структурном фазовом переходе приводит к снятию фрустраций, что способствует установлению дальнего магнитного порядка в кобальтовой подсистеме. В этом случае магнитный фазовый переход четко выражен. Структуры нестехиометрических соединений остаются неискаженными, и фрустрации в системе сохраняются. Как следствие, при понижении температуры постепенно развивается ближний магнитный порядок, для которого длина корреляции не достигает размеров кристаллитов.

Отличие между отожженным $A$ и закаленным $Q$ образцами Dу-кобальтита проявляется также на температурных зависимостях коэффициента внутреннего трения $q^{-1}(T)$ (рис. 5). Максимум поглощения для отожженного искаженного образца $A$ наблюдается вблизи температуры магнитного фазового перехода $T_{N} \sim 105 \mathrm{~K}$. Похожий максимум поглощения $q^{-1}(T)$ наблюдается также для стехиометрического образца $\mathrm{YBaCo}_{4} \mathrm{O}_{7}$ (вставка на рис. 5). Ранее было обнаружено, что этот максимум поглощения пропадает в сильно замещенных кобальтитах $R \mathrm{BaCoZn}_{3} \mathrm{O}_{7}(R=\mathrm{Y}, \mathrm{Dy}, \mathrm{Ho}, \mathrm{Er})$ [20], что подтверждает его связь с магнитным упорядочением в кобальтовой подсистеме. Асимметричная форма максимума $q^{-1}(T)$ для стехиометрического образца $A$ позволяет предположить, что он состоит из двух широких пиков, один из которых, совпадает с температурой магнитного упорядочения в кобальтовой подсистеме. Действительно, опыт показывает, что внутреннее трение вблизи фазового перехода часто обнаруживает двойной максимум поглощения, один при температуре перехода, а второй при более низкой температуре, связанный, повидимому, с релаксацией доменов. Для закаленных образцов $Q$ максимум поглощения смещен в область более низких температур и чаще всего (как для закаленного Ү-кобальтита) меньше по величине, то есть для этих образцов сохраняется только низкотемпературный максимум поглощения. Отметим дополнительно повышенное поглощение для закаленного образца Dу-кобальтита по сравнению с отожженным образцом $A$ (или широкий максимум $\left.q^{-1}(T)\right)$ в области $T \sim 280 \mathrm{~K}$. Это может быть связано с дополнительной релаксацией неупорядоченных локальных искажений для этого образца в отсутствие структурного перехода (рис. 5).

Аналогичные закономерности в поведении коэффициента внутреннего трения в зависимости от искажения структуры и содержания кислорода ранее были обнаружены для отожженного стехиометрического и закаленного образцов $\mathrm{YBaCo}_{4} \mathrm{O}_{7+x}$. Для стехиометрического искаженного образца с $x=0$ имеется большой максимум, состоящий из двух, тогда как для закаленного образца с $x \leq 0.1$ сохраняется только один низкотемпературный максимум (вставка на рис. 5).

Для кобальтитов $R \mathrm{BaCo}_{4} \mathrm{O}_{7}$ с магнитными редкоземельными ионами Но, $\mathrm{Er}$ температурные зависимости модуля Юнга и коэффициента внутреннего трения схожи с аналогичными зависимостями для $\mathrm{YBaCo}_{4} \mathrm{O}_{7}[21]$. Для искаженных и близких к стехиометрическим образцам на фоне монотонного изменения модуля Юнга в области фазового перехода $T_{N} \sim 105 \mathrm{~K}$ наблюдаются скачок $\Delta E / E_{0}$ и соответственно максимум на производной $\left(E_{0}^{-1}\right) d E / d T$. Величина скачка несколько варьируется для различных соединений $R \mathrm{BaCo}_{4} \mathrm{O}_{7+x}$ и часто проявляется только на производной $\left(E_{0}^{-1}\right) d E / d T$, которая имеет максимум, соответствующий магнитному фазовому переходу при $T_{N}$. Эти скачки сопровождаются большим максимумом на зависимости $q^{-1}(T)$ при $T_{N}$. Схожесть упругих аномалий соединений $R \mathrm{BaCo}_{4} \mathrm{O}_{7} \mathrm{c}$ ионами Y, Но, $\mathrm{Er}$ позволяет предположить, что основную роль в формировании магнитного поведения редкоземельных кобальтитов также играет кобальтовая подсистема.

\section{3. Магнитные свойства}

Для оценки взаимодействия кобальтовой и редкоземельной подсистем в $\mathrm{DyBaCo}_{4} \mathrm{O}_{7+x}$ мы провели измерения начальной магнитной восприимчивости, а также 


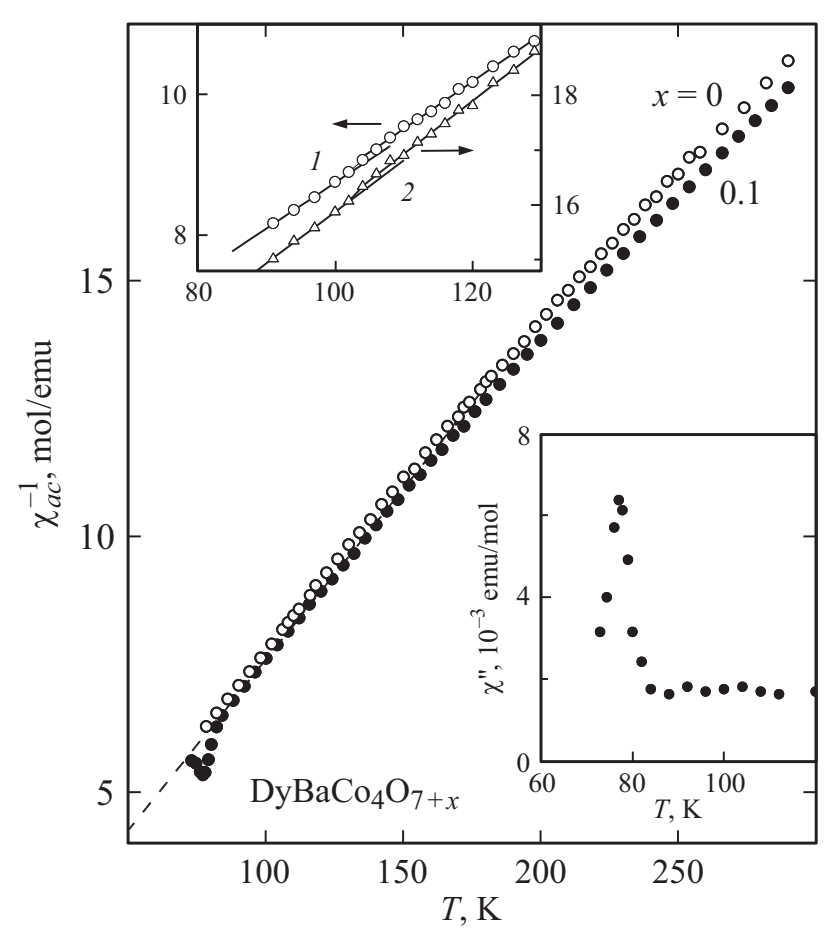

Рис. 6. Температурная зависимость обратной магнитной восприимчивости $\chi_{a c}^{-1}$ отожженных стехиометрического $(x=0$, светлые точки) и нестехиометрического $(x=0.1$, темные точки) образцов $\mathrm{DyBaCo}_{4} \mathrm{O}_{7+x}$. На нижней вставке показана мнимая часть восприимчивости нестехиометрического образца. На верхней вставке показана обратная восприимчивость стехиометрических образцов с ионами Dу (левая ось, кривая 1 ) и $\mathrm{Tm}$ (правая ось, кривая 2) в области $T_{N}$.

намагниченности в импульсном магнитном поле. На рис. 6 приведено сравнение зависимостей $\chi_{a c}^{-1}(T)$ для искаженного стехиометрического с $x=0$ (светлые точки) и неискаженного нестехиометрического с $x=0.1$ (темные точки) образцов. Видно, что в исследованной области температур восприимчивость следует закону Кюри-Вейсса, a еe величины для двух соединений очень близки при $T>100 \mathrm{~K}$, что дает близкие значения парамагнитной температуры $\theta=-14.4,-16.7 \mathrm{~K}$ и эф-

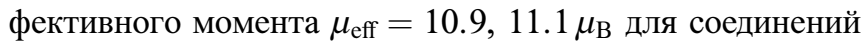
с $x=0$ и 0.1 соответственно. Величина эффективного момента близка к значению для свободного иона $\mathrm{Dy}^{3+}$ $\mu_{\mathrm{eff}}=10.6 \mu_{\mathrm{B}}$. Заметное отличие наблюдается только при температурах ниже $80 \mathrm{~K}$, где для нестехиометрического образца видны максимумы на зависимостях действительной $\chi_{a c}(T)$, и мнимой восприимчивости $\chi_{a c}^{\prime \prime}(T)$ (нижняя вставка на рис. 6). Похожие аномалии восприимчивости наблюдались ранее в работе [22] при исследовании смешанной системы $\mathrm{Dy}_{1-x} \mathrm{Ca}_{x} \mathrm{BaCo}_{4} \mathrm{O}_{7}$, где для соединения с $x=0$ также были обнаружены аномалия и максимум на кривых $\chi(T)$ и $\chi_{a c}^{\prime \prime}(T)$ соответственно. Учитывая способ получения образцов в работе [22] (закалка от высокой температуры), можно предполагать что, измерения проводились на нестехиометрическом образце $\mathrm{DyBaCo}_{4} \mathrm{O}_{7+x}$ с $x$ близком к 0.1. Такое поведение восприимчивости кобальтитов $\mathrm{DyBaCo}_{4} \mathrm{O}_{7+x}$ кажется неожиданным, поскольку выраженный магнитный переход ожидался, прежде всего, для стехиометрического образца с $x=0$.

Остается открытым вопрос, почему на зависимости $\chi_{a c}^{-1}(T)$ не обнаруживается аномалия и соответственно упорядочение в Со подсистеме при $T_{N} \sim 105 \mathrm{~K}$. Мы определили начальную восприимчивость также из линейного участка кривых намагничивания $M(H)$ в области $T_{N}$, где можно ожидать упорядочение кобальтовой подсистемы. Парамагнитная восприимчивость, полученная из кривых $M(H)$, следует закону Кюри-Вейсса с парамагнитной температурой $\theta=-14 \mathrm{~K}$, что свидетельствует о наличии антиферромагнитного обменного взаимодействия $R-$ Со (верхняя вставка на рис. 6, кривая 1). На зависимости $\chi^{-1}(T)$ можно предполагать наличие очень слабой аномалии при $T \sim 105 \mathrm{~K}$, надежное определение которой, однако, ограничено нашими экспериментальными погрешностями. В кобальтите с ионом Tm, для которого относительный вклад в полную намагниченность от Со подсистемы несколько больше (магнитный момент иона Tm меньше), эта аномалия проявляется более отчетливо (рис. 6, кривая 2).

Объяснение такого поведения на наш взгляд может быть следующим. В соединениях $R \mathrm{BaCo}_{4} \mathrm{O}_{7+x}$ с магнитными Р3-ионами доминирующим является взаимодействие в кобальтовой подсистеме, а величина обменных интегралов при замене Y на магнитный ион Dy меняется, по-видимому, не сильно, поскольку параметры решетки и углы связи также меняются не сильно. Однако вклад в магнитный момент и восприимчивость от Р3-подсистемы почти на порядок превышает вклад от Со-подсистемы. Поэтому отчетливую аномалию можно ожидать только, если при упорядочении возникает заметное эффективное поле на ионе Dу от Со-подсистемы. Это эффективное поле кроме обменного параметра

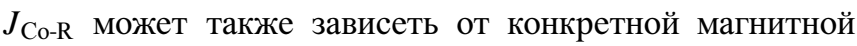
структуры упорядоченной Со-подсистемы, которая может меняться, например, за счет магнитной анизотропии.

В Ү-кобальтите, как известно, ниже $80 \mathrm{~K}$ наблюдается второй магнитный фазовый переход типа спиновой переориентации, при котором меняется антиферромагнитная магнитная структура, по-видимому, из-за магнитной анизотропии. В соединениях с магнитными Р3-ионами перехода типа спиновой переориентации ниже $80 \mathrm{~K}$ не наблюдали, хотя экспериментальные данные для Р3-кобальтитов ограничены.

Исследование намагниченности в сильных магнитных полях для Р3-кобальтитов, имеющих различные магнитные ионы, позволяет оценить вклад от отдельных подсистем. В соединении $\mathrm{DyBaCo}_{4} \mathrm{O}_{7}$ имеются две магнитные подсистемы, которые дают вклад в намагниченность и могут, вообще говоря, взаимодействовать. Ү-кобальтит позволяет изучать вклад, связанный только с кобальтовой подсистемой. 


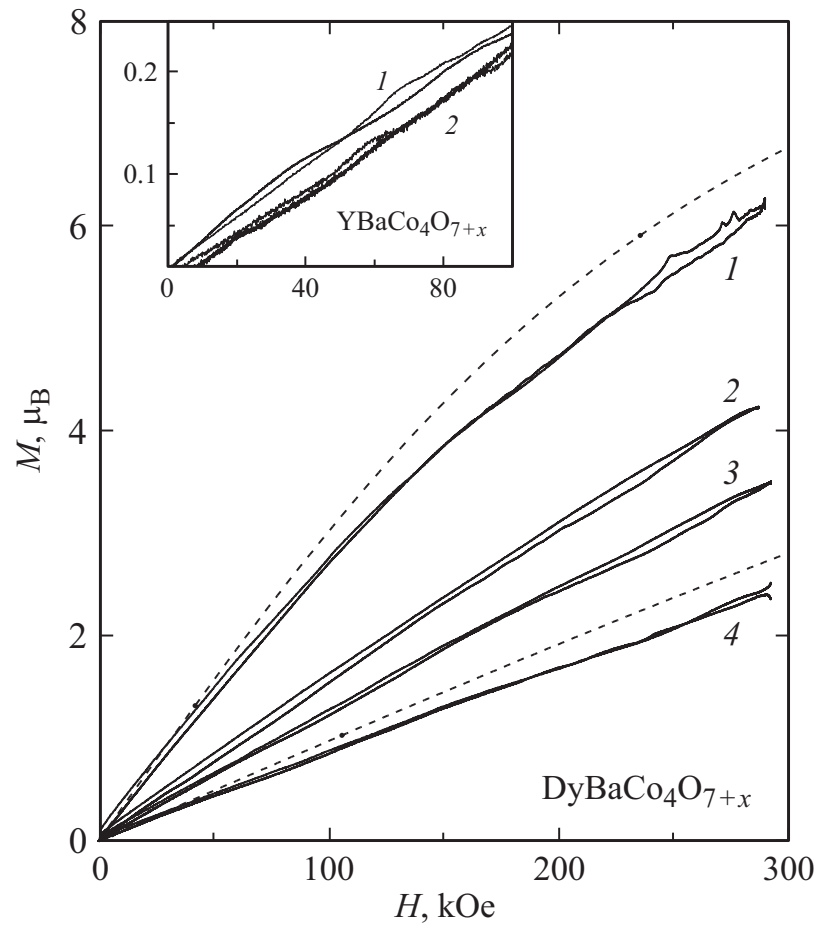

Рис. 7. Намагниченность закаленного нестехиометрического образца $\mathrm{DyBaCo}_{4} \mathrm{O}_{7+x}$ при различных температурах (кривая $1-80 \mathrm{~K}, 2-110 \mathrm{~K}, 3-160 \mathrm{~K}, 4-260 \mathrm{~K}$; пунктирные кривые - расчет по функции Бриллюэна). На вставке показана намагниченность закаленного нестехиометрического образца $\mathrm{YBaCo}_{4} \mathrm{O}_{7+x}$ при различных температурах (кривая $1-80 \mathrm{~K}$, $2-160 \mathrm{~K})$.

Сравним намагниченность кобальтитов $R \mathrm{BaCo}_{4} \mathrm{O}_{7} \mathrm{c}$ немагнитным ионом $\mathrm{Y}$ и магнитным ионом Dу. Для соединения $\mathrm{YBaCo}_{4} \mathrm{O}_{7}$ намагниченность линейна, в поле $100 \mathrm{kOe}$ не превышает $0.3 \mu \mathrm{B} / \mathrm{mol}$ и мало меняется при увеличении температуры от 80 до $210 \mathrm{~K}$ (вставка на рис. 7). Исходя из электронных конфигураций для высоко спиновых состояний ионов $\mathrm{Co}^{2+}$ и $\mathrm{Co}^{3+}$ в тетраэдрической координации и без учета орбитального вклада при параллельном упорядочении спиновых моментов трех ионов $\mathrm{Co}^{2+}\left(\mu_{i}=3 \mu_{\mathrm{B}} /\right.$ ион $)$ и одного иона $\mathrm{Co}^{3+}\left(\mu_{i}=4 \mu_{\mathrm{B}} /\right.$ ион $)$ для ферромагнитной конфигурации в сильном поле следует ожидать магнитного момента $M_{S}=13 \mu_{\mathrm{B}} / \mathrm{mol}$. Величина магнитного момента в сильном поле при низких температурах далека от этого значения, что указывает на наличие сильных антиферромагнитных взаимодействий между ионами Со.

$\mathrm{B}$ соединении $\mathrm{DyBaCo}_{4} \mathrm{O}_{C} 7$ при температуре $80 \mathrm{~K}$ и в поле $300 \mathrm{kOe}$ намагниченность достигает $\sim 6 \mu_{\mathrm{B}}$, что более чем на порядок превышает вклад Со-подсистемы (рис. 7). При повышении температуры до $260 \mathrm{~K}$ намагниченность уменьшается приблизительно в три раза, что свидетельствует о практически парамагнитном поведении редкоземельной подсистемы. Действительно зависимости $M(H)$ в области температур жидкого азота достаточно хорошо описываются функцией Бриллюэна

$$
B_{J}(x)=\frac{2 J+1}{2 J} \text { cth } \frac{(2 J+1) x}{2 J}-\frac{1}{2 J} \text { cth } \frac{x}{2 J},
$$

где $x=g_{J} \mu_{\mathrm{B}} J H / k T$, а полное квантовое число $J$ и фактор Ланде $g_{J}$ для свободного иона Dу принимают значения $J=15 / 2$ и $g_{J}=4 / 3$. Более низкое экспериментальное значение намагниченности (даже без учета вклада Со-подсистемы) по сравнению с рассчетным (пунктирные кривые на рис. 7) позволят предположить наличие небольшого антиферромагнитного эффективного поля, действующего на Р3-подсистему со стороны упорядоченной или подмагниченной Со-подсистемы. Магнитная восприимчивость, которая следует закону Кюри-Вейсса с парамагнитной температурой $\theta=-14 \mathrm{~K}$, также свидетельствует о наличии антиферромагнитного обменного взаимодействия $R-$ Со (рис. 6).

\section{4. Заключение}

В настоящей работе проведены экспериментальные исследования структурных, упругих и магнитных свойств кобальтитов $\mathrm{DyBaCo}_{4} \mathrm{O}_{7+x}$, приготовленных по различной технологии и отличающихся отклонением от стехиометрии. Для чистых Р3-кобальтитов стехиометрического состава с различными Р3-ионами наблюдаются структурные фазовые переходы, которые снимают фрустрации обменных взаимодействий. Поэтому стехиометрические образцы обнаруживают четкие магнитные фазовые переходы, сопровождаемые выраженными аномалиями физических свойств.

Наши исследования на образцах $\mathrm{YBaCo}_{4} \mathrm{O}_{7+x}$ показали, что уже при незначительном отклонении от стехиометрии по кислороду (для $x$ вблизи значения 0.1) искажение структуры пропадает. Из-за нерегулярного положения ионов избыточного кислорода в решетке структура становится дефектной, что препятствует согласованному упорядочению локальных структурных искажений по всему образцу. В неискаженной гексагональной структуре фрустрации сохраняются, развитие дальнего магнитного порядка затрудняется, и аномалии упругих характеристик при магнитном фазовом переходе быстро размываются и пропадают.

Настоящие исследования выявили заметное отличие в поведении двух близких серий $\mathrm{YBaCo}_{4} \mathrm{O}_{7+x}$ и $\mathrm{DyBaCo}_{4} \mathrm{O}_{7+x}$. Стехиометрический образец $\mathrm{DyBaCo}_{4} \mathrm{O}_{7}$ также обнаруживает искажение структуры при комнатной температуре, однако аномалия при $T_{N} \approx 105 \mathrm{~K}$ на зависимости упругого модуля $\Delta E / E(T)$ выражена слабо и при незначительном отклонении от стехиометрии $x \approx 0.1$ становятся ненаблюдаемыми. На зависимости $\chi^{-1}(T)$ стехиометрического образца аномалия при $T_{N}$ практически не наблюдается. Наоборот, на зависимости $\chi^{-1}(T)$ нестехиометрического образца при этом обнаруживается выраженная аномалия в области $T_{N 2} \sim 70 \mathrm{~K}$, совпадающей с температурой спин-пере- 
ориентационного перехода в $\mathrm{YBaCo}_{4} \mathrm{O}_{7}$. Такое поведение свидетельствует о слабой связи РЗ-подсистемы, которая остается парамагнитной при упорядочении Со-подсистемы. При наличии заметного обменного взаимодействия можно было бы ожидать возникновения эффективного обменного поля на РЗ-ионах при упорядочении Со-подсистемы и, более выраженных аномалий на зависимости $\chi^{-1}(T)$. Отметим, что магнитный Р3-ион может сильно менять магнитную анизотропию, что может сказываться на магнитной структуре в Со-подсистеме и эффективном обменном поле на Р3-ионе. Поскольку характер фазовых переходов и различные физические свойства в системе $\mathrm{DyBaCo}_{4} \mathrm{O}_{7+x}$ в очень сильной степени зависит от искажения структуры, которое определяется избытком кислорода, представляет интерес дальнейшее исследование фазообразования и фазовой диаграммы этой системы при отклонении кислорода от стехиометрии.

\section{Список литературы}

[1] L.C. Chapon, P.G. Radaelli, H. Zheng, J.F. Mitchell. Phys. Rev. B 74, 172401 (2006).

[2] P. Manuel, L.C. Chapon, P.G. Radaelli, H. Zheng, J.F. Mitchell. Phys. Rev. Lett. 103, 037202 (2009).

[3] W. Schweika, M. Valldor, P. Lemmens. Phys. Rev. Lett. 98, 067201 (2007).

[4] V. Caignaert, V. Pralong, A. Maignan, B. Raveau. Solid State Commun. 149, 453 (2009).

[5] V. Caignaert, V. Pralong, V. Hardy, C. Ritter, B. Raveau. Phys. Rev. B 81, 094417 (2010).

[6] K. Singh, V. Caignaert, L.C. Chapon, V. Pralong, B. Raveau, A. Maignan. Phys. Rev. B 86, 024410 (2012).

[7] E.A. Juarez-Arellano, A. Friedrich, D.J. Wilson L. Wiehl, W. Morgenroth, B. Winkler, M. Avdeev, R.B. Macquart, C.D. Ling. Phys. Rev. B 79, 064109 (2009).

[8] E.V. Tsipis, J.C. Waerenborgh, M. Avdeev, V.V. Kharton. J. Solidy State Chem. 182, 640 (2009).

[9] Л.П. Козеева, М.Ю. Каменева, А.И. Смоленцев, В.С. Данилович, Н.В. Подберезская. ЖСХ 6, 1108 (2008).

[10] A. Huq, J.F. Mitchell, H. Zheng, L.C. Chapon, P.G. Radaelli, K.S. Knight, P.W. Stephens. J. Solidy State Chem. 179, 1136 (2006).

[11] D.D. Khalyavin, L.C. Chapon, P.G. Radaelli, H. Zheng, J.F. Mitchell. Phys. Rev. B 80, 144107 (2009).

[12] D.D. Khalyavin, P. Manuel, B. Ouladdiaf, A. Huq, P.W. Stephens, H. Zheng, J.F. Mitchell, L.C. Chapon. Phys. Rev. B 83, 094412 (2011).

[13] M. Markina, A.N. Vasiliev, N. Nakayama, T. Mizota, Y. Yeda. J. Magn. Magn. Mater. 322, 1249 (2010).

[14] M.J.R. Hoch, P.L. Kuhns, S. Yuan, T. Besara, J.B. Whalen, T. Siegrist, A.P. Reyes, J.S. Brooks, H. Zheng, J.F. Mitchell. Phys. Rev. B 87, 064419 (2013).

[15] M. Soda, Y. Yasui, T. Moyoshi, M. Sato, N. Igawa, K. Kakurai. J. Phys. Soc. Jpn. 75, 054707 (2006).

[16] M. Valldor, Y. Sanders, W. Schweika. J. Phys.: Confer. Ser. 145, 012076 (2009).

[17] З.А. Казей, В.В. Снегирев, Л.П. Козеева, М.Ю. Каменева, А.Н. Лавров. ЖЭТФ 153, 782 (2018).
[18] Л.П. Козеева, М.Ю. Каменева, А.Н. Лавров, Н.В. Подберезская. Неорган. материалы 49, 668 ( 2013).

[19] А.В. Алексеев, М.Ю. Каменева, Л.П. Козеева, А.Н. Лавров, Н.В. Подберезская, А.И. Смоленцев, А.Н. Шмаков. Изв. РАН. Сер. физ. 77, 173 ( 2013).

[20] З.А. Казей, В.В. Снегирев, Л.П. Козеева, М.Ю. Каменева. ЖЭТФ 149, 155 (2016).

[21] Z.A. Kazei, V.V. Snegirev, A.A. Andreenko, L.P. Kozeeva, M.Y. Kameneva. Solid State Phenomena 233-234, 145 (2015).

[22] S.N. Panja, J. Kumar, S. Dengre, S. Nair. J. Phys.: Condens. Matter. 28, 486001 (2016).

Редактор Т.Н. Василевская 